\title{
Geospatial and Simulation a Point of Convergence
}

\author{
Christopher V Budas
}

Defence Science Technology Laboratory,UK, cbudas@dstl.gov.uk

Keywords: Simulation, 3D mapping, visualisation

\begin{abstract}
:
As 3D visualisation of geospatial data has become more prominent over the last decade within the Mapping and Geospatial communities, they have begun to use various techniques and processes that have previously been used by the Simulation community. In the reverse the Simulation community has progressively been making wider use of rich sources of geospatial data as the basis of terrain models. This has partly been facilitated by a continued development of processing ability of computers enabling the 3D display of geospatial data on a broader range of devices including mobile platforms.

In the past the Geospatial and Simulation communities have worked relatively independent from each other. This has subsequently led the Simulation community to manage geospatial data so that it would support their applications in the generation of terrains in both 2D and 3D.

The study will look at the two fields and how they have come together over the years. It will also consider how the evolution of standards are having an impact on the two communities and the trends that are happening within 3D mapping and simulation that are influencing and driving these changes.

Lessons learned from the Simulation community and how its work can help in a range of topics including generalisation in visualisation of data, will be given. The study will be able to offer views and perspectives of 3D visualisation in mapping presented by someone who has a Geospatial background and spent a decade using geospatial knowledge to support simulation research and has worked on generating 3D virtual representations and exploited the output for a range of purposes. Details of how the two communities can work together in the future to benefit from a mutual understanding of each other's needs and requirements will also be given.
\end{abstract}

(C) Crown copyright (2018), Dstl. This material is licensed under the terms of the Open Government Licence except where otherwise stated.

The works published in the Abstracts of the International Cartographic Association (ICA) are distributed under the Creative Commons Attribution 4.0 License. This license does not affect the Crown copyright work, which is re-usable under the Open Government Licence (OGL). The Creative Commons Attribution 4.0 License and the OGL are interoperable and do not conflict with, reduce or limit each other. 